\title{
Spin Transport and Dynamics in Multilayer Magnetic Nanostructures
}

\author{
Andrii Korostil", Mykola Krupa \\ Email address: \\ korostilandrii@gmail.com(A. Korostil) \\ ${ }^{*}$ Corresponding author
}

Department of Magnetic Mesoscopic Materials and Nanocrystalline Structures, Institute of Magnetism, Kyiv, Ukraine

To cite this article:

Andrii Korostil, Mykola Krupa. Spin Transport and Dynamics in Multilayer Magnetic Nanostructures. American Journal of Nano Research and Applications. Vol. 6, No. 1, 2018, pp. 21-66. doi: 10.11648/j.nano.20180601.13

Received: March 26, 2018; Accepted: April 13, 2018; Published: May 11, 2018

\begin{abstract}
The interconnection between the spin current and spin dynamics via the spin-dependent scattering and an accompanying by spin torque effect in ferromagnetic/normal metal based magnetic multilayer nanostructures is studied including a high fast out-of-equilibrium spin dynamics. Features of the spin transport through interfaces and its impact on spin dynamics are described on the base of the scattering matrix formalism for spin flows. The dependence of the spin torque effect on conductance character of the normal metal layers is considered. The exchange processes between the itinerant $s$ and the localized $d$ electrons are described by kinetic rate equations for electron-magnon spin-flop scattering. It is shown that the magnon distribution function remains nonthermalized on the relevant time scales of the demagnetization process, and the relaxation of the out-of-equilibrium spin accumulation among itinerant electrons provides the principal channel for dissipation of spin angular momentum from the combined electronic system.
\end{abstract}

Keywords: Magnetic Nanostructures, Spin Transport, Scattering, Spin Torque Effect, Electron-Magnon Spin-flop Scattering, Nonequilibrium Spin Dynamics

\section{Introduction}

Stacks of alternating ferromagnetic and nonmagnetic metal layers exhibit giant magnetoresistance (GMR), because their electrical resistance depends strongly on whether the moments of adjacent magnetic layers are parallel or antiparallel. This effect has allowed the development of new kinds of field-sensing and magnetic memory devices [1]. The cause of the GMR effect is that conduction electrons are scattered more strongly by a magnetic layer when their spins lie antiparallel to the layer's magnetic moment than when their spins are parallel to the moment. Devices with moments in adjacent magnetic layers aligned antiparallel thus have a larger overall resistance than when the moments are aligned parallel, giving rise to GMR. At the same time, there is the converse effect: just as the orientations of magnetic moments can affect the flow of electrons, a polarized electron current scattering from a magnetic layer can have a reciprocal effect on the moment of the layer. As shown in $[2,3]$, an electric current passing perpendicularly through a magnetic multilayer may exert a torque on the moments of the magnetic layers. This effect which is known as "spin transfer," may, at sufficiently high current densities, alter the magnetization state. It is a separate mechanism from the effects of current induced magnetic fields. Experimentally, spin-current-induced magnetic excitations such as spinwaves, [4-9] and stable magnetic reversal [6, 7] have been observed in multilayers, for current densities greater than $10^{7} \mathrm{~A} / \mathrm{cm}^{2}$.

The spin-transfer effect offers the promise of new kinds of magnetic devices and serves as a new means to excite and to probe the dynamics of magnetic moments at the nanometer scale. In order to controllably utilize these effects, however, it is necessary to achieve a better quantitative understanding of current-induced torques. A derivation of spin-transfer torques using a one-dimensional (1D) WKB approximation with spin-dependent potentials presented in [3] only take into account electrons which are either completely transmitted or 
completely reflected by the magnetic layers. For real materials the degree to which an electron is transmitted through a magnetic/nonmagnetic interface depends sensitively on the matching of the band structures across the interface $[10,11]$. It is important to incorporate such band structure information together with the effect of multiple reflections between the ferromagnetic layers, into a more quantitative theory of the torques generated by spin-transfer. This could be done using the formalism [12] based on kinetic equations for spin currents. Instead, it can be made by employ a modified Landauer-Büttiker formalism, in which the ferromagnetic layers are modeled as generalized spindependent scatterers [13]. In this case, the calculations are carried out for a quasi-one-dimensional geometry, for which formulas for the torque generated on the magnetic layers are derived when a current is applied to the system, for either ballistic or diffusive nonmagnetic layers.

Controlling spin flow in the mentioned magnetic nanostructures at ultrafast time scales using femtosecond laser pulses opens intriguing possibilities for spintronics [14]. These laser-induced perturbations $[15,16]$ stir up the most extreme regime of spin dynamics, which is governed by the highest energy scale associated with magnetic order: the microscopic spin exchange that controls the ordering temperature $T_{C}$. In contrast, at microwave frequencies the ferromagnetic dynamics in the bulk are well described by the Landau-Lifshitz-Gilbert (LLG) phenomenology [17], which has been successfully applied to the problem of the ferromagnetic resonance (FMR). At finite temperatures below $T_{C}$, the spin Seebeck and Peltier effects [18, 19] describe the coupled spin and heat currents across interfaces in magnetic heterostructures.

Despite their different appearances, the microwave, thermal and ultrafast spin dynamics are all rooted in the exchange interactions between electrons. It is thus natural to try to advance a microscopic describing of the ultrafast dynamics based on the established phenomena at lower energies. Although some attempts have been made $[20,21]$ to extend the LLG phenomenology to describe ultrafast demagnetization in bulk ferromagnets, no firm connection exists between the ultrafast spin generation at interfaces and the microwave spin-transfer and spin-pumping effects [22] or the thermal spin Seebeck and Peltier effects.

Solving the above-mentioned problem involves unification of the energy regimes of microwave, thermal, and ultrafast spin dynamics in magnetic heterostructures from a common microscopic point of view, so that the parameters that control the high and low energy limits of spin relaxation originate from the same electron-magnon interactions [23]. In addition to the unified framework, these unique contributions are the history-dependent, nonthermalized magnon distribution function and the crucial role of the out-of-equilibrium spin accumulation among itinerant electrons as the bottleneck that limits the dissipation of spin angular momentum from the combined electronic system.

The paper is organized as follows. In Sec. 2, the interconnection between the spin current and spin dynamics via the spin-dependent scattering and an accompanying spin torque effect in ferromagnetic $(F)$ /normal metal $(N)$ based magnetic multilayer nanostructure is studied. Section 3 is devoted to description of the impact of electron-magnon spin-flop scattering on out-of-equilibrium spin dynamics in $F / N$ based magnetic nanostructures.

\section{Spin-Dependent Interface Scattering-Induced Torques in Magnetic Multilayer Nanostructures}

\subsection{Features of Spin Transfer Effect}

In this section, a simple intuitive picture of the physics behind the spin-transfer effect is considered. The connection between current-induced spin-transfer torques and the spindependent scattering that occurs when electrons pass through a magnetic-nonmagnetic interface can be illustrated most simply by considering the case of a spin-polarized current incident perpendicularly on a single thin ferromagnetic layer $F$, as shown in Figure 1. The layer lies in the $y-z$ plane, with its magnetic moment uniformly pointed in the $z$ direction, and it is assumed that the current is spin-polarized in the $z-x$ plane at an angle $\theta$ to the layer moments. The incoming electrons can therefore be considered as a coherent linear superposition of basis states with spin in the $+z$ direction (amplitude $\cos \theta / 2$ ) and $-z$ direction (amplitude $\sin \theta / 2)$. At first, it is assumed that the layer is a perfect spin filter, so that spins aligned with the layer moments are completely transmitted through the layer, while spins aligned antiparallel to the layer moment are completely reflected. For incident spins polarized at an angle $\theta$, the average outgoing current will have the relative weights $\cos ^{2} \theta / 2$ polarized in the $+z$ direction and transmitted to the right and $\sin ^{2} \theta / 2$ polarized in the $-z$ direction and reflected to the left. Consequently, both of the outgoing electron spin fluxes (transmitted and reflected) lie along the $z$ axis, while the incoming (incident) electron flux has a component perpendicular to the magnetization, along the $x$ axis, with magnitude proportional to $\sin \theta$. This $x$ component of angular momentum must be absorbed by the layer in the process of filtering the spins.

Because the spin-filtering is ultimately governed by the $s-d$ exchange interaction between the conduction electrons and the magnetic moments of the layer, the angular momentum is imparted to the layer moments and produces a torque on them. This exchange torque [24], which is proportional to the electron current through the layer and to $\sin \theta$, is in the direction to align the moments with the polarization of the incident spin current. The symmetry of this model precludes any generation of torque from the spin filtering of a current of unpolarized electrons. To generate the effect, then, a second ferromagnetic layer is needed to first spin polarize the current (Figure 2). In that case, spin angular momentum is transferred from one layer to the flowing electrons and then from the electrons to the second layer. 


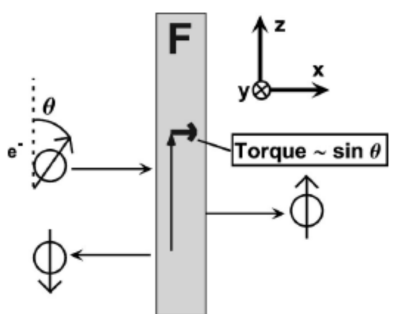

Figure 1. Schematic of exchange torque generated by spin filtering. Spinpolarized electrons are incident perpendicularly on a thin ideal ferromagnetic layer. Spin filtering removes the component of spin angular momentum perpendicular to the layer moments from the current; this is absorbed by the moments themselves, generating an effective torque on the layer moments.
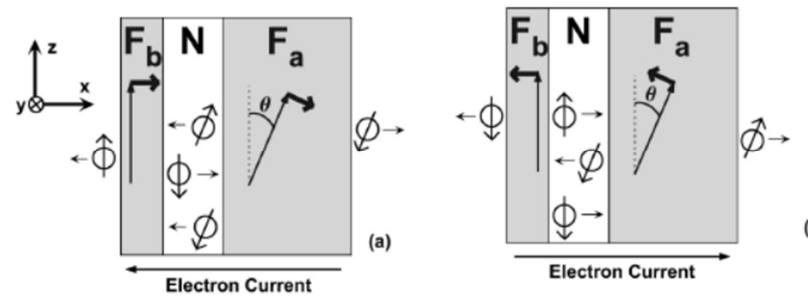

Figure 2. Qualitative picture of asymmetry of spin-transfer torque with respect to current bias in a F//NF junction. For left-going electrons (a), initially polarized by a magnetic layer $F_{a}$, the moments of layer $F_{b}$ experience a torque so as to align them with layer $F_{a}$. The electron current reflected from layer $F_{b}$, in turn, exerts a torque on layer $F_{a}$ so as to antialign it with the moment of layer $F_{b}$. Subsequent reflections between the layers reduce but do not eliminate this torque. If the current is reversed (b) the overall sign of the torque is reversed, encouraging the moment of layer $F_{b}$ to align antiparallel with layer $F_{a}$

The presence of this second layer has the additional effect of allowing for multiple scattering of the electrons between the two layers, which gives rise to an explicit asymmetry of the torque with respect to current direction. This asymmetry is an important signature which can be used to distinguish spin-transfer-induced torques from the torques produced by current-generated magnetic fields. To see how the asymmetry arises, consider the ferromagnet-normal-metal- ferromagnet $(F / N / F)$ junction shown in Figure 2. It consists of two ferromagnetic layers $F_{a}$ and $F_{b}$, with moments pointing in directions $m_{a}$ and $m_{b}$, separated by a normal metal spacer $N$. Normal metal leads on either side of the trilayer inject an initially unpolarized current into the system. When the current enters the sample from the left (Figure 2(a)), electrons transmitted through $F_{a}$ will be polarized along $m_{a}$. As long as the normal metal spacer is smaller than the spin-diffusion length $(100 \mathrm{~nm}$ for $\mathrm{Cu})$, this current will remain spin-polarized when it impinges on $F_{b}$ and will exert a torque on the moment of $F_{b}$ in a direction so as to align $m_{b}$ with $m_{a}$.

Repeating the argument for $F_{b}$, we find that the spin of the electrons reflected from layer $F_{b}$ is aligned antiparallel to $m_{b}$, and, hence in turn, exerts a torque on the moment of $F_{a}$ trying to align $m_{a}$ antiparallel with $m_{b}$. Subsequent multiple reflections of electrons between $F_{a}$ and $F_{b}$ can serve to reduce the magnitudes of the initial torques, but they do not eliminate or reverse them, as the electron flux is reduced upon each reflection. If there were no anisotropy forces in the sample, the net result would be a motion with both moments rotating in the same direction (clockwise in Figure 2(a)), as described previously in [3]. When the current is injected from the right, the directions of the torques are reversed. The flow of electrons exerts a torque on $F_{a}$ trying to align its moment parallel with $m_{b}$, while it exerts a torque on $F_{b}$ so as to force the moment in layer $F_{b}$ antiparallel with $m_{a}$.

In $[3,6]$, the layer $F_{a}$ was taken to be much thicker than $F_{b}$, so that intralayer exchange and anisotropy forces will hold the orientation of $m_{a}$ fixed. In that case, one is only interested in the torque on $F_{b}$, which serves to align $m_{b}$ either parallel or antiparallel with the fixed moment $m_{a}$ depending on the current direction. This asymmetric current response has been employed in both a point-contact geometry [7] and in a thin-film pillar geometry 8 to switch the moments in $F / N / F$ trilayers from a parallel to an antiparallel configuration by a current pulse in one direction, and then from antiparallel to parallel by a reversed current. For weakly interacting layers, either orientation can be stable in the absence of an applied current, so that the resistance versus current characteristic is hysteretic, and the devices can function as simple current-controlled memory elements.

Often, the transport properties of magnetic multilayers are described using "two-current" models [8], in which one assumes that the effects of spin-polarized currents can be described completely in terms of incoherent currents of spinup and spin-down electrons. Normally, only the cases of purely parallel or purely antiparallel magnetic layers are considered, and the spin currents are conserved upon passing through each normal-metal-ferromagnet interface.

In the mentioned case there can be no current-induced torque on either magnetic layer. It is important to recognize that such two-current models are not appropriate to calculate current-induced torques for the more general case of arbitrary tilt angle between the moments in a magnetic multilayer, as the simple example discussed in this section demonstrates. Tilting of the spin axis is an essential point of the physics [9], and this must be described in terms of a coherent sum of spin-up and spin-down basis states. In the general case, the spin flux is not conserved upon passing through a magnetic layer, so that a torque is applied to each magnetic layer. As it turned out, this is a simple consequence of different transmission amplitudes for the spin-up and spin-down components of the electron flux.

\subsection{Spin Flux and Torque in the Scattering Approach}

Treating the ferromagnetic layers as perfect spin filters 
provides important qualitative insights into spin transfer, but for a complete qualitative and quantitative picture, a more general approach is required. This can be realized by introduction a scattering matrix description of the $\mathrm{F} / \mathrm{N} / \mathrm{F}$ junction which allows to deal with nonideal (magnetic and nonmagnetic) layers. It is important to relate the torque $\tau_{b}$ exerted on layer $F_{b}$ by an unpolarized incident electron beam to the scattering properties of the layers. Although restriction by formulas to the $F / \mathrm{N} / F$ junction (see Figure 3 ), the mentioned method is applicable for an arbitrary array of magnetic-nonmagnetic layers.

The spin flux $\boldsymbol{J}$ In the $x$ direction (the direction of current flow) can be written in the form [13]

$$
\boldsymbol{J}(x)=\frac{\hbar^{2}}{2 m} \operatorname{Im} \int d y d z\left(\phi^{\dagger}(x) \boldsymbol{\sigma} \phi(x)\right)
$$

where $\phi(x)$ is a spinor wave function and $\sigma$ is the vector of Pauli matrices

$$
\phi(x)=\left(\begin{array}{l}
\phi_{\uparrow}(x) \\
\phi_{\downarrow}(x)
\end{array}\right), \quad \boldsymbol{\sigma}=\left(\begin{array}{c}
\sigma_{x} \\
\sigma_{y} \\
\sigma_{z}
\end{array}\right) .
$$

Note that no local equation of conservation can be written for the spin flux, since in general, the Hamiltonian (of the itinerant electrons) does not conserve spin. Specifically, the magnetic layers can act as sources and sinks of spin flux, so that the spin flux on different sides of a $F$ layer can be different. When the angle $\theta$ is 0 or $\pi$ (typical situation for GMR), the commutativity between the Hamiltonian and the (electron) spins is restored (in the absence of spin-flip scattering). It should be stressed that the Hamiltonian of the full system (electrons plus local moments of the ferromagnets plus the environment) does commute with the total spin. Therefore the spin lost by the itinerant electrons has to be gained by the other parts of the system.

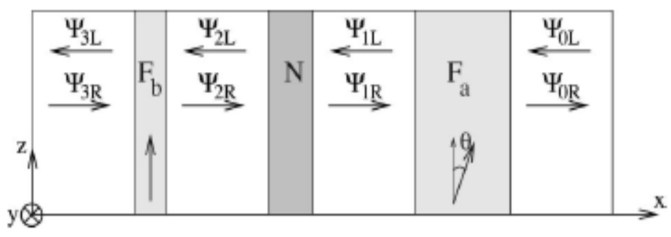

Figure 3. Schematic of the setup used for the definition of the scattering matrices of the $F$ and $N$ layers. The two layers $F_{b}$ and $F_{a}$ are ferromagnetic layers whose magnetic moment is oriented as shown in the bottom of the figure. The layer $N$ is a nonmagnetic metal spacer. Amplitude of left and right moving propagating waves are defined in fictitious ideal leads 0, 1, 2, and 3 between the layers and between the layers and the reservoirs.

Figure 3 shows the $F / N / F$ junction where (fictitious) perfect leads (labeled $0,1,2$, and 3 ) have been added in between the layers $F$ and $N$ and between the $F$ layers and the electron reservoirs on either side of the sample. The introduction of these leads allows for a description of the system using scattering matrices. In the perfect leads, the transverse degree of freedom is quantized, giving $N_{\text {ch }}$ propagating modes at the Fermi level, where $N_{\mathrm{ch}} \sim A / \lambda_{F}^{2}, A$ being the cross section area of the junction and $\lambda_{F}$ the Fermi wave length. Expanding the electronic wave function in these modes, we can describe the system in terms of the projection $\Psi_{i, \mathrm{j}}$ of the wave function onto the left $(j=1)$ or right $(j=2)$ going modes in the region $i=(0,1,2,3)$. The $\Psi_{i j}$ is $2 N_{\mathrm{ch}}$ component vector, counting the $N_{\mathrm{ch}}$ transverse modes and spin. The amplitudes of the wave function in two neighboring ideal leads are connected through the scattering matrices $S_{1}=S_{a}, S_{2}=S_{N}$ and $S_{3}=S_{b}$ that relate amplitudes of outgoing modes and incoming modes at the layer (see [25]) by the relation.

$$
\left(\begin{array}{c}
\Psi_{i, 1} \\
\Psi_{i-1,2}
\end{array}\right)=S_{i}\left(\begin{array}{c}
\Psi_{i, 2} \\
\Psi_{i-1,1}
\end{array}\right), \quad i=(1,2,3)
$$

The scattering matrix $S_{i}$ is $4 N_{\mathrm{ch}} \times 4 N_{\mathrm{ch}}$ unitary matrices. The generalized matrix $S_{i}$ is decomposed into $2 N_{\text {ch }} \times 2 N_{\text {ch }}$ reflection and transmission matrices.

$$
S_{i}=\left(\begin{array}{cc}
r_{i} & t^{\prime} \\
t_{i} & r^{\prime}{ }_{i}
\end{array}\right)
$$

Normalization is done in such a way that each mode carries unit current. Due to the spin degree of freedom, $r_{i}=\left\|r_{i, \sigma \sigma^{\prime}}\right\|$ and $\sigma, \sigma^{\prime}=(\uparrow, \downarrow)$, where the reflection and transmission matrices can be written in terms of four $N_{\text {ch }} \times N_{\text {ch }}$ blocks: where the subscripts $\uparrow, \downarrow$ refer to spin up and down in the $z$-axis basis. The scattering matrix of the magnetic layers depends on the angle $\theta$ the moments may make with the $z$ axis. The matrix $S_{i}(\theta)$ is related to $S_{i}(0)$ through a rotation in spin space:

$$
\begin{aligned}
& r_{i}(\theta)=R_{\theta} r_{i}(0) R_{-\theta}, \quad r_{i}^{\prime}(\theta)=R_{\theta} r_{i}^{\prime}(0) R_{-\theta}, \\
& t_{i}(\theta)=R_{\theta} t_{i}(0) R_{-\theta}, \quad t^{\prime}{ }_{i}(\theta)=R_{\theta} t^{\prime}{ }_{i}(0) R_{-\theta}
\end{aligned}
$$

where

$$
R_{\theta}=\left(\begin{array}{rr}
\cos \frac{\theta}{2} & -\sin \frac{\theta}{2} \\
\sin \frac{\theta}{2} & \cos \frac{\theta}{2}
\end{array}\right) \otimes 1_{N}
$$

The nonmagnetic metallic layer will not affect the spin states, i.e., $r_{N \uparrow \downarrow}=r_{N \downarrow \uparrow}=0$ and $r_{N \uparrow \uparrow}=r_{N \downarrow \downarrow}$. We need to keep track of the amplitudes within the system in order to calculate the net spin flux deposited into each magnetic layer. Therefore, we define $2 N_{\text {ch }} \times 2 N_{\text {ch }}$ matrices $\Gamma_{i j}$ and $\Lambda_{i j}$ ( $i=(0,1,2,3))$ so that all the $\Psi_{i, \mathrm{j}}$ can be expressed as a function of the amplitudes incident from the two electrodes (regions 0 and 3 ):

$$
\left(\begin{array}{l}
\Psi_{i, 1} \\
\Psi_{i, 2}
\end{array}\right)=\left(\begin{array}{cc}
\Gamma_{i 1} & \Lambda_{i 1} \\
\Gamma_{i 2} & \Lambda_{i 2}
\end{array}\right)\left(\begin{array}{l}
\Psi_{0,1} \\
\Psi_{3,1}
\end{array}\right)
$$


with the convention that $\Gamma_{01}=\Lambda_{32}=1$ and $\Gamma_{32}=\Lambda_{01}=0$. In order to calculate the torque exercised on layer $\mathrm{F}_{b}$ for a current entering from the left, we need the matrix $\Gamma_{21}$, which for simplify is denoted as $\Omega\left(\Omega=\Gamma_{21}\right)$. The matrix $\Omega$ relates the amplitudes $\Psi_{21}$ to the incoming amplitudes $\Psi_{01}$. Putting that $\Psi_{32}=0$ and using (2), the matrix of the equation (6) can be explicitly expressed via elements of the scattering matrices $S_{i}$. Consequently, the following expression can be obtained for the matrix $\Omega$ :

$$
\Omega=\frac{1}{1-r_{n} r_{b}^{\prime}} t^{\prime}{ }_{n} \frac{1}{1-r_{a} t_{n} r_{b}^{\prime}\left(1-t_{n} r_{b}^{\prime}\right) t_{n}^{\prime}-t_{a} r_{n}^{\prime}} t_{a}^{\prime}
$$

which will enter in determination of the torque on the moment of the ferromagnetic layer $F_{b}$.

If the system is connected to two unpollarized electron reservoirs on its two sides, then in equilibrium, the modes in the reservoirs are filled up to the Fermi level $\varepsilon_{F}$. The spin current through the system is generated when the chemical potential in the left (right) reservoir is slightly increase by $\delta \mu_{3}\left(\delta \mu_{0}\right)$. The spin current $J_{i}$ in each region $i=0,1,2,3$ is the difference of the left going and right going contributions. In according to (1) and (6).

$$
\begin{aligned}
& \frac{\partial J_{i}}{\partial \mu_{0}}=\frac{1}{4 \pi} \operatorname{Re}\left[\operatorname{Tr} \sigma \Gamma_{i R} \Gamma_{i R}^{\dagger}-\operatorname{Tr} \sigma \Gamma_{i R} \Gamma_{i R}^{\dagger}\right] \\
& \frac{\partial J_{i}}{\partial \mu_{3}}=\frac{1}{4 \pi} \operatorname{Re}\left[\operatorname{Tr} \sigma \Lambda_{i L} \Lambda_{i L}^{\dagger}-\operatorname{Tr} \sigma \Lambda_{i L} \Lambda_{i L}^{\dagger}\right] .
\end{aligned}
$$

Since the spin flux on both sides of $F_{b}$ is different, angular momentum has been deposited in the $F_{b}$. This creates a torque $\tau_{b}=J_{3}-J_{2}$ on the moment of the ferromagnet. Setting $\delta \mu_{0}=e V_{0}$ gives

$$
\frac{\partial r_{b}}{\partial V_{0}}=-\frac{e}{4 \pi} \operatorname{Re}_{2 N_{c h}}\left[\Sigma \Omega \Omega^{\dagger}\right], \Sigma=\sigma-t^{\prime \prime}{ }_{b} \sigma t^{\prime}{ }_{b}-r^{\prime \dagger}{ }_{b} \sigma r_{b}^{\prime}
$$

This equation can be simplified further if the spin-transfer effect is due entirely to spin filtering (as argued in [3]) as opposed to spin-flip scattering of electrons from the magnetic layers. That is at $\tau_{b \uparrow \downarrow}=\tau_{b \downarrow \uparrow}=\tau_{a \uparrow \downarrow}(\theta=0)=\tau_{a \downarrow \uparrow}(\theta=0)=0$, then

$$
\begin{aligned}
\frac{\partial r_{b}}{\partial V_{0}} & =-\frac{e}{4 \pi} \operatorname{Re}_{T_{N_{c h}}}\left[\left(\Omega_{\uparrow \uparrow} \Omega_{\downarrow \uparrow}^{\dagger}+\Omega_{\uparrow \downarrow} \Omega_{\downarrow \downarrow}^{\dagger}\right)\right. \\
& \left.\times\left(1-r_{b \uparrow \uparrow}^{\prime} r_{b \downarrow \downarrow}^{\prime \dagger}-r_{b \uparrow \uparrow}^{\prime} r_{b \downarrow \downarrow}^{\prime \dagger}\right)\right],
\end{aligned}
$$

where off-diagonal spin-flip terms are related to spin-flip scattering both in normal and magnetic layers. There is no spin flux conservation in this system, $\partial J_{i} / \partial \mu_{3}$ can be different from $\partial J_{i} / \partial \mu_{0}$ and, hence, there can be a nonzero spin flux even when the chemical potentials are identical in the two reservoirs.

The existence of a zero-bias spin flux and the resulting torques reflect the well-known itinerant-electron-mediated exchange interaction (also known as the RKKY interaction) between two ferromagnetic films separated by a normalmetal spacer. This interaction can in fact be described within a scattering framework [26-29]. The zero-bias torque has to be added to the finite-bias contribution (given by (11)). Since the former is typically a factor $N_{c h}^{-1}$ smaller and vanishes upon ensemble averaging [25]. Therefore, the zero-bias contribution to the torque can be neglected compared to the bias induced torque, for which $\partial r_{b} / \partial V_{0}=\partial r_{b} / \partial V_{3}$.

\subsection{Averaging over the Normal Metal Layer}

The torque on the moments of the ferromagnetic layers $F_{a}$ and $F_{b}$ not only depends on the scattering matrices $S_{a}$ and $S_{b}$ of these layers, but also of the scattering matrix $S_{N}$ of the normal metal layer in between. If the normal layer is disordered, $\tau_{a}$ and $\tau_{b}$ depend on the location of the impurities; if $N$ is ballistic the torque depend sensitively on the electronic phase shift accumulated in $N$. In general, sample to sample fluctuations of the torque will be a factor $N_{c h}^{-1}$ smaller than the average [25]. Hence, if $N_{c h}$ is large, the torque is well characterized by its average. After averaging, the zero-bias spin transfer current, corresponding to the RKKY interaction described above, vanishes, and only the torque caused by the electron current remains. Because all effects of quantum interference in the $N$ layer will disappear in the process of averaging, the derived results are unchanged if the reflection and transmission matrices include processes in which the energy of the electron changes during scattering [30].

\subsubsection{The Torque for Disordered Normal Metal Layers}

The scattering matrix of the normal layer can be written using the standard polar decomposition [25]

$$
S_{a}=\left(\begin{array}{cc}
U & 0 \\
0 & V^{\prime}
\end{array}\right)\left(\begin{array}{cc}
\sqrt{1-T} & i \sqrt{T} \\
i \sqrt{T} & \sqrt{1-T}
\end{array}\right)\left(\begin{array}{cc}
U^{\prime} & 0 \\
0 & V
\end{array}\right)
$$

where $U, V, U^{\prime}$ and $V^{\prime}$ are $2 N_{c h} \times 2 N_{c h}$ unitary matrices and $T$ is a diagonal matrix containing the eigenvalues of $t_{n} t_{n}^{\dagger}$. Since $S_{n}$ is diagonal in spin space, matrices we find that $U, U^{\prime}, V$ and $V^{\prime}$ are block diagonal: $U\left(U^{\prime}\right)=\left(\begin{array}{cc}u\left(u^{\prime}\right) & 0 \\ 0 & u\left(u^{\prime}\right)\end{array}\right)$ and similar definitions for $v$ and $v^{\prime}$. The outer matrices in (12) thus mix the modes in a ergodic way while the central matrix contains the transmission properties of the layer, which determine the average conductance of $N$.

It is necessary to average (11) over both the unitary matrices and $T$. A diagrammatic technique for such averages has already been developed in [31] and can be used to calculate $\partial \rho_{b} / \partial V_{0}$ in leading order in $1 / N_{c h}$. It is a general property of such averages that the fluctuations are a factor of order $N_{c h}$ 
smaller than the average. This justifies the statement above, that the ensemble averaged torque is sufficient to characterize the torque exerted on a single sample.

The resulting expression for $\partial \rho_{b} / \partial V_{0}$ can be written in a form very similar to the one for (11) if one uses a notation that involves $4 \times 4$ matrices. Then, the average over the transmission eigenvalues $T$ can be obtained taking into account that the average of a function is the function of the average, to leading order in $1 / N_{c h}$. Thus the average over $T$ amounts to the replacement

$$
\hat{t}_{n}=\frac{g_{N}}{N_{c h}} 1_{4}, \quad \hat{r}_{n}=\left(1-\frac{g_{N}}{N_{c h}}\right) 1_{4},
$$

where $g_{N}$ is the conductance of the normal layer and $1_{4}$ is the $4 \times 4$ unit matrix. Entering the $4 \times 4$ block matrix $\hat{\Sigma}$ with the first and fourth rows equal to $\left(\begin{array}{llll}\Sigma_{\uparrow \uparrow} & \Sigma_{\uparrow \downarrow} & \Sigma_{\downarrow \uparrow} & \Sigma_{\downarrow \downarrow}\end{array}\right)$ and the zero second and third rows, it can be obtained that

$$
\left\langle\frac{\partial r_{b}}{\partial V_{0}}\right\rangle=-\frac{e}{4 \pi} \operatorname{Re} \operatorname{Tr}_{4}\left[\widehat{\Sigma} \Omega^{\dagger}\right]
$$

where $\hat{\Sigma}$ coincide with $\Omega$ after the formal replacements $r_{n} \rightarrow \hat{r}_{n n}$ and $t_{n} \rightarrow \hat{t}_{n}$. In the absence of spin-flip scattering (14) reduces to

$$
\begin{aligned}
& \left\langle\frac{\partial r_{b}}{\partial V_{0}}\right\rangle=-\frac{e}{2 \pi} \operatorname{Re}\left[\left(\widehat{\Omega}_{3,1}+\widehat{\Omega}_{3,4}\right)\right. \\
& \left.\times \operatorname{Tr}_{N_{c h}}\left(1-r_{b \uparrow \uparrow}^{\prime}{ }_{b \downarrow \downarrow \downarrow}^{\prime \dagger}-t^{\prime}{ }_{b \uparrow \uparrow t_{b \downarrow \downarrow}^{\prime \dagger}}\right)\right]
\end{aligned}
$$

The same formalism can be used to calculate the conductance $g$ of the system using the Landauer formula. One gets $\langle g\rangle=\left(N_{c h} / h\right) \times\left[\hat{t}^{\prime}{ }_{1,1}+\hat{t}_{1,4}^{\prime}+\hat{t}_{4,1}^{\prime}+\hat{t}_{4,4}^{\prime}\right]$, where $t^{\prime}$ being the total matrix $t^{\prime}=t^{\prime}{ }_{b} \Omega$.

It would like to note that, while our theory started from a fully phase coherent description of the $\mathrm{F} / \mathrm{N} / \mathrm{F}$ trilayer, including the full $4 N_{c h} \times 4 N_{c h}$ scattering matrices of the $F N$ interfaces, the final result can be formulated in term of $2 \times 4$ parameters, represented by the matrices $\hat{r}_{a}$ and $\hat{r}_{b}^{\prime}(2 \times 16$ parameters in the case of spin-flip scattering). This confirms the statement that for a diffusive normal-metal spacer all effects of quantum interferences are washed out [25].

The torque is characterized by symmetry properties. Due to the conservation of current, the total torque deposited on the full system is antisymmetric with respect to current direction and the equation

$$
\partial \tau_{b} / \partial V_{0}+\partial \tau_{a} / \partial V_{0}=-\left[\partial \tau_{b} / \partial V_{3}++\partial \tau_{a} / \partial V_{3}\right]
$$

must be held before averaging. The averaging results in $\left\langle\partial \tau_{b} / \partial V_{0}\right\rangle=\left\langle\partial \tau_{b} / \partial V_{3}\right\rangle$. Thus, for $N_{c h} \gg 1$, the linear response of the torque to a small bias voltage is described by the expression.

$$
\tau_{\beta}=\left\langle\frac{\partial \tau_{b}}{\partial V_{0}}\right\rangle\left(V_{0}-V_{3}\right)
$$

In the given geometry, where $F_{a}$ and $F_{b}$ are in the $x-z$ plane, the only nonzero component of the torque is $\tau_{b}^{x}$. The torque vanishes when the moments are completely aligned or antialigned (all the matrices are diagonal in spin space and therefore no $x$ component of the spin can be found). Around these two limits, the torque is symmetric in respect to the angle $\theta \rightarrow-\theta$ and $\pi-\theta \rightarrow \pi+\theta)$. There is no symmetry between $\theta$ and $\pi-\theta$. In addition, the two layers are not equivalent and exchanging the scattering matrices of $F_{a}$ and $F_{b}$ also changes the torque.

The equation (14) can be simplified in some particular cases. In the case of ideal spin filter, so that majority (minority) spins are totally transmitted (reflected) by either layer, it reduces to

$$
\left\langle\frac{\partial r_{b}^{x}}{\partial V_{0}}\right\rangle=-\frac{e}{2 \pi} \frac{g_{N} \sin \theta}{3+\cos \theta}=-\frac{h}{4 \pi e}\langle g\rangle \frac{\tan \theta / 2}{2},
$$

where $\langle g\rangle=4\left(e^{2} / h\right) g_{N} \cos ^{2} \theta /(3+\cos \theta) \quad$ is the average magnetoconductance. As expected, for left-going electrons $\left(V_{0}<0\right)$ the torque is positive, so it acts to align the moment of the magnetic layer $F_{b}$ toward the one of $F_{a}$.

In the considered case of weak $s$ - $d$ exchange coupling, i.e., when the scattering coefficients depend only weakly on spin, with no spin-flip scattering in the ferromagnetic layers, $g_{a}$ and $g_{b}$ can be defined as the average conductance per spin of the two layers (in unit of $e^{2} / h$ ). Then, the conductance of $F_{a}$ alone is $g_{a}+\delta g_{a}$ and $g_{a}-\delta g_{a}$ for, respectively, the majority and minority spins, which defines the spin scattering asymmetry $\delta g_{a}$. In that case, in lowest nontrivial order in $\delta g_{a}$ and $\delta g_{b}$ :

$$
\begin{gathered}
\left\langle\frac{\partial \tau_{b}^{x}}{\partial V_{0}}\right\rangle=\frac{e}{2 \pi} \frac{\sin \theta}{2\left(1-g_{b} / N_{c h}\right)} \\
\times \frac{g_{N}^{2} \delta g_{a} \delta g_{b}^{2}}{\left(g_{a} g_{b}+g_{N}\left(g_{a}+g_{b}-2 g_{a} g_{b} / N_{c h}\right)^{2}\right.} .
\end{gathered}
$$

This formula shows that the torque is always non zero for arbitrary small spin scattering asymmetry. This proves the statement, that multiple reflections between the $F$ layers, fully taken into account here, cannot completely eliminate the torque. The torque is not symmetric with respect to interchanging the layer $F_{a}$ and $F_{b}$, in contrast to the conductance. If one changes $\delta g_{a}$ to $-\delta g_{a}$, the sign of the torque is reversed. However, $\partial \tau_{b}^{x} / \partial V_{0} \propto \delta g_{b}^{2}$, so if one changes $\delta g_{b}$ to $-\delta g_{b}$, the sign of the torque is unchanged. The sign of the torque on a ferromagnetic layer therefore depends on whether the other layer is a positive or negative polarizer, but not on the sign of filtering for the layer 
experiencing the torque. This is true also in the general case. The quantity $g_{N}^{2}$ appears through its square. Indeed, in order for some spin to be deposited in the layer $F_{b}$, some left going electrons have to be reflected by $F_{b}$ and exit the system from the right hand side. Therefore these electrons cross the normal layer at least twice and this leads to the factor $g_{N}^{2}$. On the other hand the conductance is linear in $g_{N}$. Therefore in order to maximize the torque deposited per current, one has to use the cleanest possible normal metal spacer. This statement is true in this limit of weak filtering, but not in general. Note that in the previous case (perfect spin filtering) the torque is proportional to $g_{N}$ instead of the expected $g_{N}^{2}$. Indeed, in that case, once the electron has been reflected by the layer $F_{b}$, it cannot go through $F_{a}$ which works as a perfect wall for it. Therefore current conservation implies that it goes out of the system through the right. For $g_{N} \ll N_{c h}$, the torque is actually proportional to $g_{N}^{2}$ for arbitrary spin asymmetry (except perfect filtering), and one gets

$$
\left\langle\frac{\partial \tau_{b}^{x}}{\partial V_{0}}\right\rangle \propto g_{N}^{2} \sin \theta, \quad g_{N} \ll N_{c h}
$$

where the factor of proportionality being a complicated function of the transmission robabilities of the layers.

\subsection{Current-Driven Magnetic Switching}

Application to current-driven magnetic switching involves calculation of torques for scattering parameters which are more appropriate for the transition metal trilayers. In this case, the torque per unit current $I, \tau_{b}^{x} / I=\left\langle(1 / g) \times \partial \tau_{b}^{x} / \partial V\right\rangle$.

The main features of the mentioned system are that the $\theta$ dependence of the torque is not of a simple $\sin \theta$ form, and that the torque per unit current diverges at $\theta=0$.

The main feature of this system is that the $\theta$ dependence of the torque is not of a simple $\sin \theta$ form, and that the torque per unit current diverges at $\theta=0$. In the imperfect case, when one of the layer $\left(F_{b}\right)$ is a nearly perfect polarizer while the other one is not, the character behavior of the torque is represented in Figure 4.

As can be seen in Figure 4 on the left, although the divergence at $\theta=\pi$ is regularized, $\tau_{b}^{x} / I$ remains sharply peaked near $\theta=\pi$. This is relevant for the critical current needed to switch the magnetization of $F_{b}$ from $\theta=\pi$ to $\theta=0$. The switching of the domains follows from a competition between the spin-transfer torques on the one hand and restoring forces from local fields, anisotropy, exchange coupling, etc.

The competition between these forces has been considered phenomenologically [7, 32] using a Landau-Lifschitz-Gilbert equation. The torques for $\theta$ close to 0 and $\pi$ determine the critical currents to overturn a metastable parallel (antiparallel) alignment of the moment in $F_{a}$ and $F_{b}$. Hence the critical current should be different at $\theta=0$ and $\theta=\pi$.

Features of the dependence of the derivative of the torque (with respect to $\theta$ closely to 0 ) on the conductance of the normal layer $g_{N}$ in the same system with one perfectly polarizing $F$ layer and one partially polarizing layer, is represented in Figure 4 on the right.
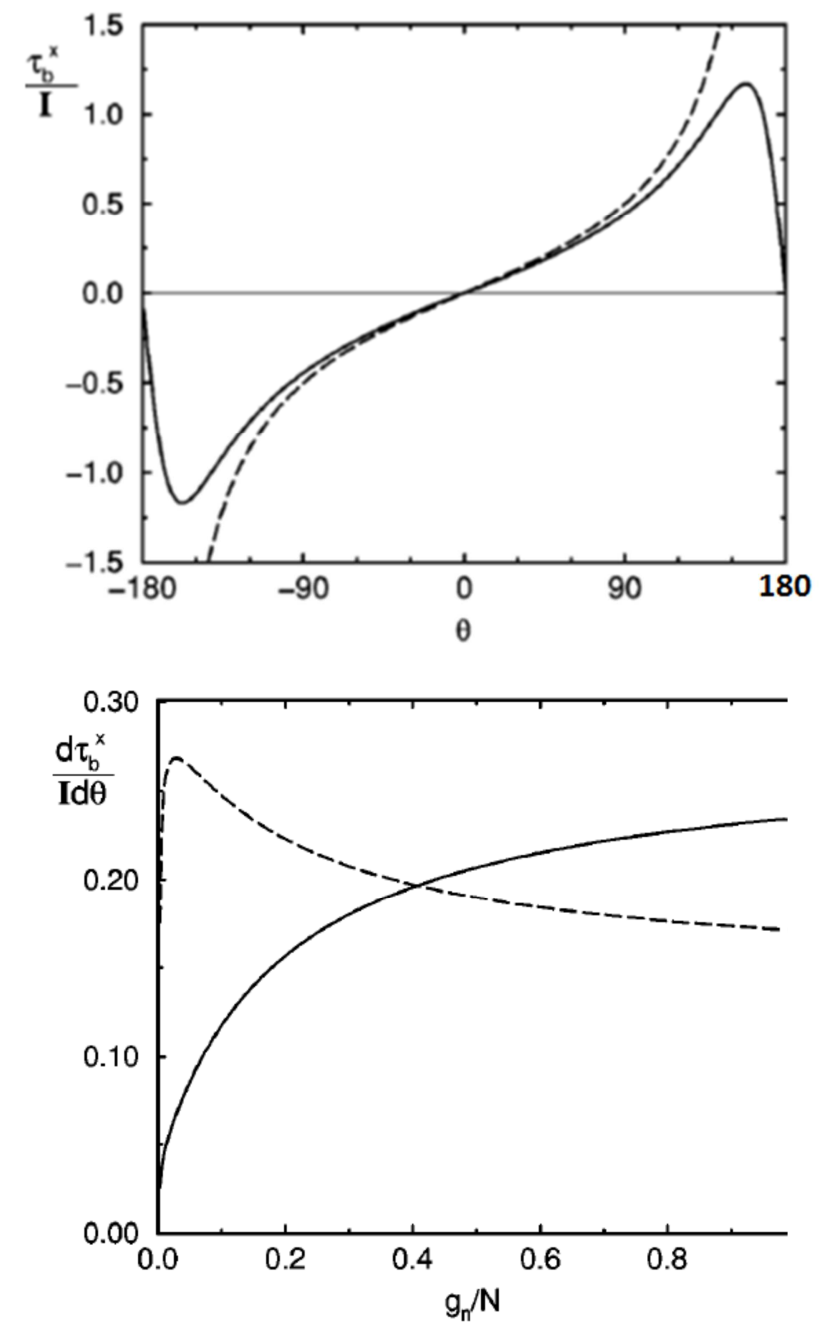

Figure 4. The normalized torque as a function of $\theta$ (on the left) and its derivative as a function the normalized conductance $g_{N}$ at $\theta=0$ (on the right) per unit current for the case, where $F_{b}$ is a nearly perfect polarizer $\left(\left|t_{b \uparrow}\right|^{2}=0.999,\left|t_{b \downarrow}\right|^{2}=0.001\right.$ and $F_{a}$ is not $\left(\left|t_{a \uparrow}\right|^{2}=0.3,\left|t_{a \uparrow}\right|^{2}=0.01\right)$ (solid line). On the left, the case of perfect polarizers is described by the equation (17) (dashed line). On the right, the dashed line corresponds to the case, when $F_{a}$ is a nearly perfect polarizer and $F_{b}$ is not.

Switching the two layers has a drastic effect on the torque, even at a qualitative level. In the case where $F_{a}$ is the nearly perfect layer (dashed line), a maximum of the torque is found for $g_{N} / N_{c h} \ll 1$, i.e., in that case, a dirty metal spacer would give a higher torque (per unit of current) than a clean one.

In the above mentioned description, the scattering matrices of the ferromagnetic layers appear as free input parameters. However, it can be calculated from first principle calculations for specific materials. Such an approach has been taken in 
$[10,11]$ and the results can be used to give some estimates of torques that can be expected in realistic systems.

\section{Out-of-Equilibrium Spin Dynamics in F/N Based Structures}

\subsection{Features of Out-of-Equilibrium Spin Dynamics}

The first reports on ultrafast demagnetization in $\mathrm{Ni}$ [33] challenged the conventional view of low-frequency magnetization dynamics at temperatures well below $T_{C}$. A multitude of mechanisms and scenarios have been suggested to explain the observed quenching of the magnetic moment. Some advocate direct coherent spin transfer induced by the irradiating laser light as the source of demagnetization [34]. Alternative theories argue that ultrafast spin dynamics arise indirectly through incoherent heat transfer to the electron system [35]. Recent experiments have demonstrated that nonlocal laser irradiation also induces ultrafast demagnetization, and atomistic modeling supports the view that heating of magnetic materials is sufficient to induce ultrafast spin dynamics [16, 36]

Terahertz (THz) magnon excitations in metallic ferromagnets have recently been proposed as an important element of ultrafast demagnetization [37, 38]. The elementary interaction that describes these excitations is the electron-magnon scattering. The proposed approach is based on kinetic equations, which were used for the low-frequency spin and charge transport associated with the microwave magnetization dynamics in heterostructures [39] and with the linear spin-caloritronic response [19, 40]. One treats farfrom-equilibrium spin dynamics, in which transport is dominated by magnons and hot electrons. Electron-magnon scattering plays a critical role in this regime. Description of this interaction is related to the transverse spin diffusion in the bulk and the spin-mixing physics, e.g., spin transfer and spin pumping [40], at the interfaces.

\subsection{A Model of the Out-of-Equilibrium Ultra-Fast Spin Dynamics}

Characteristic properties of the out-of-equilibrium ultrafast spin dynamics are described by the quantum-mechanical model bilayer system comprising ferromagnetic $(F)$ and normal metal layers, in which the localized spins are distinct from the itinerant electrons at the energy scales of interest. According to the accepted description of relaxation in ferromagnetic metals, the loss of energy and angular momentum from localized $d$ electrons is mediated by the exchange interaction to the itinerant $s$ electrons. The spin transfer from $d$ to $s$ states is accompanied by the relaxation of the $s$ electron spins to the lattice through an incoherent spinflip process caused by the spin-orbit coupling.

Ultra-fast spin dynamics in bulk ferromagnetic metal is described by the quantum kinetic equations. The $F \mid N$ interfacial spin transport due to electron-magnon interactions follows a similar essential structure, unifying the bulk and interfacial spin dynamics in magnetic heterostructures. The
Hamiltonian that describes $F$ is $H=H_{0}+H_{s d}$, where $H_{0}$ consists of decoupled $s$ - and $d$ - electron energies, including the kinetic energy of the itinerant electron bath, the $d-d$ exchange energy, dipolar interactions, and the crystalline and Zeeman fields. The $s-d$ interaction is

$$
H_{s d}=J_{s d} \sum_{j} \boldsymbol{S}_{j}^{d} s\left(r_{j}\right)
$$

where $J_{s d}$ is the exchange energy and $\boldsymbol{S}_{j}^{d}\left(\boldsymbol{s}\left(\boldsymbol{r}_{j}\right)\right)$ is the $d$ electron ( $s$-electron) spin vector (spin density) at lattice point $j$. The $s-d$ interaction can be expressed in terms of bosonic and fermionic creation and annihilation operators:

$$
H_{s d}=\sum_{q k k^{\prime}} V_{q k k^{\prime}} a_{q} c_{k \uparrow}^{\dagger} c_{k^{\prime} \downarrow}+\text { H.c. }
$$

where $a_{q}^{\dagger}\left(a_{q}\right)$ is the Holstein-Primakoff creation (annihilation) operator for magnons with wave number $q$ and $c_{k \sigma}^{\dagger}\left(c_{k \sigma}\right)$ is the creation (annihilation) operator for $s$ electrons with momentum $k$ and spin $\sigma . H_{s d}$ describes how an electron flips its spin while creating or annihilating a magnon with momentum $q$ and spin $\sigma$. The scattering strength is determined by the matrix element $V_{q k k}$.

In (21), terms of the form $a_{q}^{\dagger} a_{q^{\prime}} c_{k \sigma}^{\dagger} c_{k^{\prime} \sigma}$, which describe multiple-magnon scattering and do not contribute to a net change in magnetization along the spin-quantization axis have disregarded. It also is disregarded higher-order terms associated with the Holstein-Primakoff expansion. Fully addressing magnonic correlation effects in the ultrafast regime would require a rigorous approach, e.g., using nonequilibrium Keldysh formalism [41]. However, when the $s$ - $d$ coupling (20) is not the dominant contribution to $H$, a mean-field approach and Fermi's golden rule were used to compute the spin transfer between the $s$ and $d$ subsystems. Additionally, it is assumed that all relevant energy scales are much smaller than the Fermi energy $\varepsilon=k_{B} T_{F}$ of the itinerant $s$ electrons. In this limit, the electronic continuum remains largely degenerate, with electron-hole pairs present predominantly in the vicinity of the Fermi level.

In the given bilayer system, localized spin density points in the negative $z$ direction at equilibrium, with saturation value $S$ (in units of $\hbar$ ). In the presence of a magnon density $n_{d}$, the longitudinal spin density becomes $S_{z}=n_{d}-S$. The magnons are assumed to follow a quadratic dispersion relation $\varepsilon_{q}=\hbar \omega=\varepsilon_{0}+A q^{2}$, where $\varepsilon_{0}$ is the magnon gap and $A$ parametrizes the stiffness of the ferromagnet. $\left\langle a_{q}^{\dagger} a_{q^{\prime}}\right\rangle=n\left(\varepsilon_{q}\right) \delta_{q q^{\prime}}$ defines the magnon distribution function $n\left(\varepsilon_{q}\right)$, which is related to the total magnon density through $n_{d}=\int_{\varepsilon_{0}}^{\varepsilon_{b}} d \varepsilon_{q} D\left(\varepsilon_{q}\right) n\left(\varepsilon_{q}\right)$, where $D\left(\varepsilon_{q}\right)=\sqrt{\varepsilon_{q}-\varepsilon_{0}} /\left(4 \pi^{2} A^{3 / 2}\right)$ is the magnon density of states. The integral over $D\left(\varepsilon_{q}\right)$ is cut off at an energy corresponding to the bandwidth, $\varepsilon_{b}=k_{B} T_{C}$, which is the magnon energy at the edge of the Brillouin zone. 
Because of the $s$ - $d$ interaction (20), the itinerant $s$ electrons have a finite spin density at equilibrium (see Figure 5). One of the key driving forces of the out-of-equilibrium spin dynamics is the spin accumulation $\mu_{s}=\delta \mu_{\uparrow}-\delta \mu_{\downarrow}$. The bands for spin-up and spin-down electrons are split by $\Delta_{x c} \sim J_{s d} S a^{3}$, where $a$ is the lattice constant of $F$. Introducing a dynamical exchange splitting results in the relation $\mu_{s}=\delta n_{s} / D-\Delta_{x c}$ [42], where $\delta n_{s}$ is the out-of-equilibrium spin density of the $s$ electrons, $D=2 D_{\uparrow} D_{\downarrow} /\left(D_{\uparrow}+D_{\downarrow}\right)$, and $D_{\uparrow(\downarrow)}$ is the density of states for spin-up (spin-down) electrons at the Fermi level. Because the mean-field band splitting due to the $s$ - $d$ exchange vanishes when the $d$ orbitals are fully depolarized, $\delta \Delta_{x c} / \Delta_{x c}= \pm n_{d} / S$, where the sign determines whether the $s$ and $d$ orbitals couple ferromagnetically (-) or antiferromagnetically $(+)$.

The rate of spin transfer (per unit volume) between the $s$ and $d$ subsystems due to electron-magnon spin-flop processes is determined from (21) by Fermi's golden rule [40]:

$$
I_{s d}=\int_{\varepsilon_{a}}^{\varepsilon_{b}} d \varepsilon_{q} \Gamma\left(\varepsilon_{q}\right)\left(\varepsilon_{q}-\mu_{s}\right) D\left(\varepsilon_{q}\right)\left[n_{B E}\left(\varepsilon_{q}-\mu_{s}\right)-n\left(\varepsilon_{q}\right)\right]
$$

where $\Gamma\left(\varepsilon_{q}\right)$ parametrizes the scattering rate at energy $\varepsilon_{q}$. In the derivation of (22) it have assumed that the kinetic energy of the itinerant electrons and the empty states (holes) thermalize rapidly due to Coulombic scattering and that they are distributed according to Fermi-Dirac statistics. Correspondingly, it can be shown that the electron-hole pairs follow the Bose-Einstein (BE) distribution function, $n_{B E}\left(\varepsilon_{q}-\mu_{s}\right)=\left\{\exp \left[\beta_{s}\left(\varepsilon_{q}-\mu_{s}\right)\right]-1\right\}^{-1}$ at the electron temperature $T_{s}=1 /\left(k_{B} \beta_{s}\right)$. The number of available scattering states is influenced by the spin accumulation $\mu_{s}$. (a)

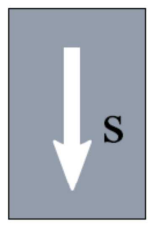

(b)

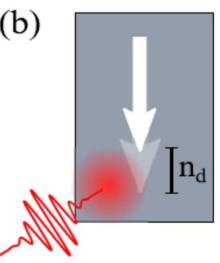

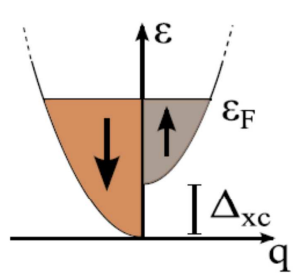

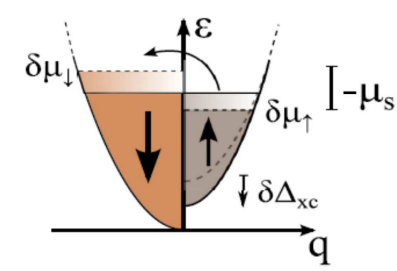

Figure 5. (a) Sketch of the density of s electron states in a ferromagnetic metal with saturation spin density $S$. At equilibrium, the exchange splitting $\Delta_{x c}$ shifts the bands for spin-up and spin-down electrons. (b) A laser pulse heats the $s$ electron bath. The out-of-equilibrium spin accumulation $\mu_{s}=\delta \mu_{\uparrow}-\delta \mu_{\downarrow}$ results from two different mechanisms: electron-magnon scattering induces a spin density among the s electrons, and the meanfield exchange splitting is shifted by $\delta \Delta_{x c}$ by the induced magnon density $n_{d}$.
In contrast to the low-energy treatment in [40], the derivation of (22) does not constrict the form of the magnonic distribution $n\left(\varepsilon_{q}\right)$ to the thermalized $\mathrm{BE}$ distribution function. When the time scale of the $s$ - $d$ scattering is faster than the typical rates associated with magnon-magnon interactions, magnons are not internally equilibrated shortly after rapid heating of the electron bath, as also predicted by atomistic modeling [43]. Consequently, the occupation of the magnon states can deviate significantly from the $\mathrm{BE}$ distribution on the time scale of the demagnetization process. Such treatment differs from that, in which the excited magnons are assumed to be instantly thermalized with an effective spin temperature and zero chemical potential and the thermally activated electron bath is assumed to be unpolarized.

\subsection{Heat Pulse-Induced Out-of-Equilibrium Spin Dynamics}

The $s$ - $d$ scattering rate can be phenomenologically expanded as $\Gamma\left(\varepsilon_{q}\right)=\Gamma_{0}-\chi\left(\varepsilon_{q}-\varepsilon_{0}\right)$, where $\Gamma_{0}$ (which vanishes in the simplest Stoner limit parametrizes the scattering rate of the long-wavelength magnons and $\chi\left(\varepsilon_{q}-\varepsilon_{0}\right) \propto q^{2}$ describes the enhanced scattering of higherenergy magnons due to transverse spin diffusion [44]. In general, one might expect other terms of higher order in $q$ to be present in this expansion as well. The quantity $\Gamma\left(\varepsilon_{q}\right)$ is extrapolated up to the bandwidth $\varepsilon_{b}$ that should be sufficient for qualitative purposes. Neglecting any direct relaxation of magnons to the static lattice or its vibrations (i.e., phonons), $\partial_{t} n_{d}=I_{s d} / \hbar$. The equations of motion for the $s$-electron spin accumulation and the $d$-electron magnon distribution function are

$$
\begin{gathered}
\partial_{t} \mu_{s}=-\frac{\mu_{s}}{\tau_{s}}+\frac{\rho}{\hbar} I_{s d} \\
\partial_{t} n\left(\varepsilon_{q}\right)=\frac{\Gamma\left(\varepsilon_{q}\right)}{\hbar}\left(\varepsilon_{q}-\mu_{s}\right)\left[n_{B E}\left(\varepsilon_{q}-\mu_{s}\right)-n\left(\varepsilon_{q}\right)\right]
\end{gathered}
$$

where $\rho$ determines the feedback of the demagnetization on $\mu_{s}$ and $\tau_{s}$ is the spin-orbit relaxation time for the $s$ electron spin density relaxing to the lattice. Here, $\tau_{s}$ is typically on the order of picoseconds [45] and represents the main channel for the dissipation of angular momentum out of the combined electronic system. In general, $\tau_{s}$ also depends on the kinetic energy of the hot electrons after laser-pulse excitation. However, it is assumed that $\tau_{s}$ is independent of energy. $\rho=\rho_{D}+\rho_{\Delta}=-1 / D+\Delta_{x c} / S$ includes effects arising from both the out-of-equilibrium spin density and the dynamic exchange splitting. For ferromagnetic $(-) s-d$ coupling, these effects add up, whereas for antiferromagnetic (+) coupling, they compete.

At low temperatures, low-frequency excitations result in purely transverse spin dynamics. In the classical picture of rigid magnetic precession, the transverse relaxation time $\tau_{2}$ 
is determined by the longitudinal relaxation time $\tau_{1}$ via the relation, $1 / \tau_{2}=1 /\left(2 \tau_{1}\right)=\alpha \omega$, where $\alpha$ is the Gilbert damping parameter and $\omega$ is the precession frequency. Indeed, in the $\operatorname{limit}\left(q, T_{s}\right) \rightarrow 0 \partial_{t} n_{d} \rightarrow(\Gamma / \hbar) \varepsilon_{0} n_{d}$, which is identical to the LLG phenomenology, indicating that $\varepsilon_{0}=\hbar \omega$ and thus $\Gamma_{0}=2 \alpha$. This result establishes the important link between the scattering rate $\Gamma_{0}$ in this treatment and the Gilbert damping parameter that is accessible through FMR experiments.

In the opposite high-frequency limit, pertinent to ultrafast demagnetization experiments, the layer $F$ to be in a low temperature equilibrium state before being excited by a $\mathrm{THz}$ laser pulse at $t=0$, upon which the effective temperature of the itinerant electron bath instantly increases such that $T_{s} \geq T_{C}$. This regime is clearly beyond the validity of the LLG phenomenology, which is designed to address the low energy extremum of magnetization dynamics. Dissipation in the LLG equation, including relaxation terms based on the

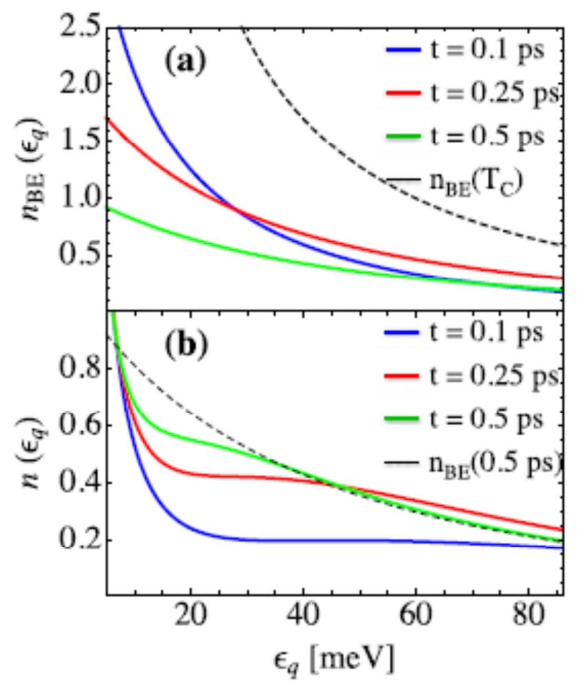

stochastic Landau-Lifshitz-Bloch treatment [46], is subject to a simple Markovian environment without any feedback or internal dynamics. This perspective must be refined for high frequencies when no subsystem can be viewed as a featureless reservoir for energy and angular momentum.

The nonthermalized nature of the excited magnons can be appreciated in the limit in which $\mu_{s}$ is small compared with $\varepsilon_{0}$ and no magnons are excited $\left(n\left(\varepsilon_{0}=0\right)\right)$ for $t<0$. After rapid heating of the itinerant electrons at $t=0$, the time evolution of the magnonic distribution follows

$$
n\left(\varepsilon_{q}, t\right) \approx n_{B E}\left(\varepsilon_{q}, t\right)\left[1-\exp \left(\Gamma\left(\varepsilon_{q}, t / \hbar\right)\right]\right.
$$

This result implies that, initially, the high-energy states are populated much faster than low-energy states. When $\mu_{s}$ becomes sizable, the coupled equations (23) and (24) must be solved subject to a suitable $T_{s}(t)$. Figure $6(\mathrm{a}, \mathrm{b})$ presents numerical solutions of (23) and

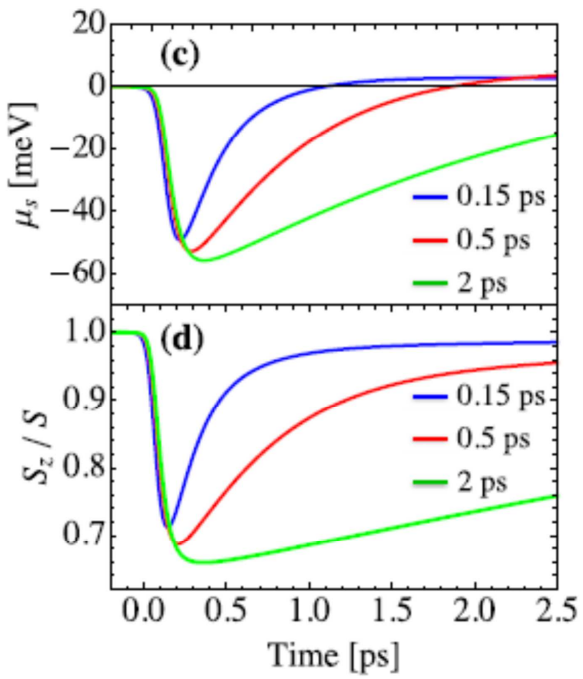

Figure 6. Numerical solutions of the system (32) and (33) after $T_{s}$ is increased from $10^{2}$ to $10^{3} \mathrm{~K}\left(T_{C}\right)$ within $50 f_{s}$ with a decay time of 2 ps. $\varepsilon_{0}=5$ meV, $A=0.6 \mathrm{meV} / \mathrm{nm}^{2}, \rho=6 \mathrm{meV} / \mathrm{nm}^{3}, \tau_{s}=2 \mathrm{ps}$, and $\alpha^{*}=10 \alpha=0.1$. (a) The itinerant electron-hole pair distribution $n_{B E}\left(\varepsilon-\mu_{s}\right)$ is rapidly depleted by the spin accumulation $\mu_{s}$ that is built up via electron-magnon scattering. (b) In the magnon distribution $n\left(\varepsilon_{q}\right)$ the high-energy magnon states are rapidly populated, whereas the low-energy states remain unaffected on short time scales. (c) Time evolution of the spin accumulation $\mu_{s}(t)$ and (d) the longitudinal spin density $-S_{z}(t)$ with different decay times of $T_{s}: 0.15,0.5$, and $2 \mathrm{ps}$.

(24) when $T_{s}$ is increased from $10^{2}$ to $10^{3} \mathrm{~K}$ within $50 \mathrm{fs}$ with a decay time of 2 ps. By comparison, internal magnonmagnon interactions equilibrate the distribution function on the time scale $\tau_{e q}^{-1} \sim \hbar^{-1} \varepsilon_{m}\left(\varepsilon_{m} /\left(k_{B} T_{C}\right)\right)^{3}$ [40], where $\varepsilon_{m}$ is a characteristic energy of the thermal magnon cloud. For short times, $I_{s d}$ (see (22)) dominates the magnon dynamics, and the magnon population is significantly differ from the thermalized BE distribution.

When $T_{s}>T_{C}$, the thermally excited electron-hole pairs are populated in accordance with the classical Rayleigh-Jeans distribution, $n_{B E}\left(\varepsilon_{q}-\mu_{s}\right) \rightarrow k_{B} T_{s} /\left(\varepsilon_{q}-\mu_{s}\right)$. Assuming, for simplicity, that the expansion for $\Gamma\left(\varepsilon_{q}\right)$ is valid throughout the Brillouin zone, (22) yields

$$
\left.\partial_{t} n_{d}\right|_{t \rightarrow 0}=I_{s}(0) / \hbar=\left(\Gamma_{0}+3 \chi\left(\varepsilon_{b}-\varepsilon_{0}\right) / 5\right) k_{B} T_{s} / \hbar
$$

Thus, the demagnetization rate is initially proportional to the temperature of the electron bath but is reduced by the lack of available scattering states for high energy magnons within the time scale of the demagnetization process. Figure $6(\mathrm{c}, \mathrm{d})$ illustrates the time evolution of the out-of-equilibrium spin accumulation $\mu_{s}(t)$ and the longitudinal spin density $-S_{z}(t)$ for different decay times of $T_{s}$.

In the ultrafast regime, the electron-magnon spin-flop scattering is governed by the effective Gilbert damping 
parameter $\alpha^{*}=\chi\left(\varepsilon_{b}-\varepsilon_{0}\right)$. Experimental investigations of the magnon relaxation rates on $\mathrm{Co}$ and $\mathrm{Fe}$ surfaces confirm that high- $q$ magnons have significantly shorter lifetimes than low$q$ magnons [38]. It is reasonable to assume that the same effects are also present in the bulk. The initial relaxation time scale in the ultrafast regime is $\tau_{i} \sim\left(\alpha * \hbar^{-1} k_{B} T_{s}\right)^{-1}$. This generalizes the result of [20] for the ultrafast relaxation of the longitudinal magnetization to arbitrary $\alpha^{*}$ based on the transverse spin diffusion [44].

The notion of magnons becomes questionable when the intrinsic linewidth approaches the magnon energy, which corresponds to $\alpha^{*} \sim 1$.

Staying well below this limit and consistent with [40], it is assumed that $\alpha^{*}=0.1$. For $T_{C}=10^{3} \mathrm{~K}$ the initial relaxation time scale $\tau_{i} \sim 10^{2}\left(T_{C} / T_{S}\right)$ fs, which is generally consistent with the demagnetization rates observed for ultrafast demagnetization in Fe [47].

It can be shown that the interfacial scattering follows a structure similar to that of the bulk scattering in a unified description based on the electron-magnon interaction. Figure 7 presents a schematic illustration of an $F \mid N$ interface. In magnetic heterostructures and for stand-alone ferromagnets on a conducting substrate, the demagnetization dynamics of $F$ are also affected by the spin accumulation in $N \mu_{N}(x)$, which can impact how nonlocal laser irradiation (e.g., the heating of $\mathrm{N}$ alone) induces ultrafast demagnetization of $F$ [15]. By adding terms of the form

(1)

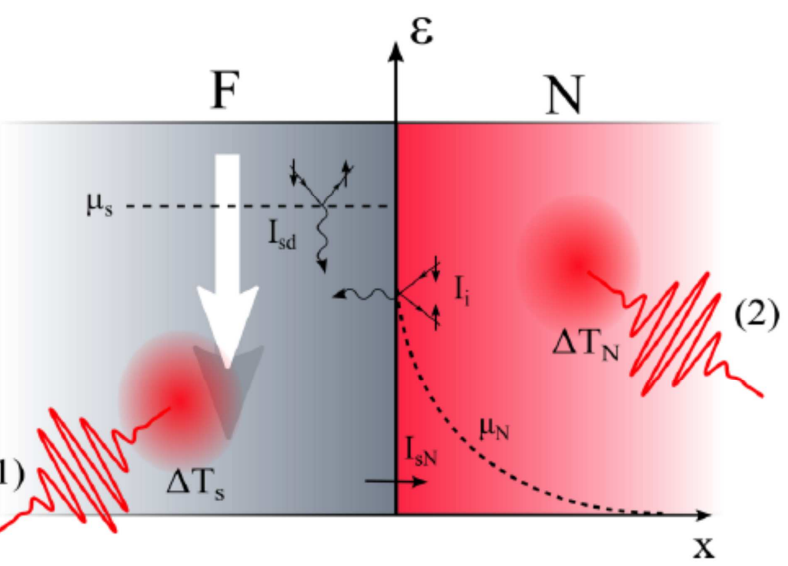

Figure 7. Sketch of a metallic ferromagnet $(F)$ coupled to a normal metal $(N)$. In the ultrafast regime, both the rapid heating of s electrons in $F$ by $\Delta T_{s}$

(labeled (1)\} and the heating of $N$ by $\Delta T_{N}$ (labeled (2)) can demagnetize $F$. $I_{\text {sd }}$ (see (31)) induces the spin accumulation $\mu_{s}$ in $F$, whereas $I_{i}$ (8) induces the spin accumulation $\mu_{N}^{0}$ at the $F \mid N$ interface. Subsequently, $\mu_{N}(x)$ diffuses into $N$ until it vanishes due to spin-flip dissipation to the lattice. The additional interfacial spin current $I_{s N}$, due to the thermodynamic biases $\delta \mu=\mu_{s}-\mu_{N}^{0}$ and $\delta T=T_{s}-T_{N}$, can be described by conventional thermoelectric parameters for longitudinal spin-dependent transport.

$\sim \sum_{q k k^{\prime}} U_{q k k^{\prime}} a_{q} c_{k \uparrow}^{\dagger} c_{k^{\prime} \downarrow}$ to $H_{s d}$, where $c_{k \uparrow}^{\dagger}\left(c_{k^{\prime} \downarrow}\right)$ describes the creation (annihilation) of an electron with spin up (down) at the $\mathrm{F} \mid \mathrm{N}$ interface, the interfacial spin transfer (per unit area) due to electron-magnon spin-flop scattering is [40]

$$
I_{i}=\int_{\varepsilon_{0}}^{\varepsilon_{b}} d \varepsilon_{q} \Gamma^{i}\left(\varepsilon_{q}\right)\left(\varepsilon_{q}-\mu_{N}^{0}\right) D\left(\varepsilon_{q}\right)\left[n_{B E}\left(\varepsilon_{q}-\mu_{N}^{0}\right)-n\left(\varepsilon_{q}\right)\right]
$$

where $\mu_{N}^{0}=\mu_{N}(0)$ is the spin accumulation at the interface and $\Gamma^{i}\left(\varepsilon_{q}\right)$ parametrizes the interfacial scattering rate.

The scattering of coherent long-wavelength magnons at the $F \mid N$ interface can be described in the language of spin pumping/spin Seebeck effects [40], parametrized by the spin mixing conductance $g_{\uparrow \downarrow}$ (per unit area) [39]. Motivated by $\Gamma\left(\varepsilon_{q}\right)$ in the bulk, the interfacial scattering rate can be written as $\Gamma^{i}\left(\varepsilon_{q}\right)=g_{\uparrow \downarrow}^{*}\left(\varepsilon_{q}\right) /(\pi S)$, where $g_{\uparrow \downarrow}^{*}$ reduces to $g_{\uparrow \downarrow}$ for low energy scattering, $\varepsilon_{q} \rightarrow \varepsilon_{0}$. The interface scattering (see (26)) dominates the microwave spin relaxation in thin ferromagnetic layers of thickness $d_{F} \leq 10 \mathrm{~nm}[39,48]$. This trend should continue for higher frequencies and is relevant for ultrafast spin dynamics in thin magnetic layers in heterostructures.

The energy dependence of the effective spin-mixing conductance to be relatively weak compared to that of the bulk scattering $\Gamma\left(\varepsilon_{q}\right)$, which can be severely constrained at low energies due to momentum conservation. For a finite temperature bias $\delta T$ across the interface and for magnons thermalized at the temperature $T<T_{C}$, the connection to the thermal spin Seebeck and Peltier effects is made by identifying $S=\partial_{t} I_{i}$ and $\Pi=T S$ [49] as the Seebeck and Peltier coefficients, respectively.

\section{Conclusions}

The spin-transfer-induced torques on the magnetic moments of $F / N / F$ systems caused by a flowing current has described in the framework of the scattering matrix formalism. This description deals with the effects of multiple scattering between the layers using the scattering matrices of the $F \mid N$ interfaces as input parameters. Both the cases of a diffusive and ballistic normal metal spacer are considered. In the diffusive case, the high-dimensional scattering matrices of the $F \mid N$ interfaces only appear through the averaging over the normal metal layers. This allows to make qualitative predictions about the role of the interface transparency, normal metal resistance, etc., without detailed knowledge of the microscopic details of the system.

The description has focused on the effects of "spin filtering" as the mechanism for current-induced torque, i.e., the difference in the transmission and reflection probabilities for electrons with spins parallel and antiparallel to the moments of the ferromagnetic layers (the diagonal terms in the matrices for the reflection and transmission amplitudes). A different source of spin-dependent scattering related to spin-flip scattering is described by the off diagonal terms in the scattering matrices. Its effect can be twofold. In the normal 
spacer, it would decrease the effective polarization, and therefore the torque. However, in the ferromagnet, the rate of spin-flip scattering is symmetric with respect to minority and majority spins, and therefore spin-flip scattering may also be an additional source of torque.

The concepts of transverse spin diffusion in bulk ferromagnets and the interfacial spin-mixing physics have extended to address the ultrafast spin dynamics observed in rapidly heated magnetic heterostructures. In the ultrafast regime, the relative importance of the bulk scattering and the interfacial scattering can be extracted from measurements of demagnetization strength and spin currents in magnetic heterostructures. For metallic ferromagnets in the bulk, treating the magnonic subsystems as quasiequilibrated and parametrized by an effective temperature is insufficient to describe the farfrom-equilibrium spin dynamics induced by pulsed laser heating The magnon distribution function remains nonthermalized on the relevant time scale of the demagnetization process, in which the relaxation of the out-of-equilibrium spin accumulation limits the dissipation of spin angular momentum from the combined electronic system.

\section{References}

[1] J. Akerman, "Toward a Universal Memory," Science, vol. 308, pp. 508-510, April 2005.

[2] L. Berger, "Emmision of spin waves by a magnetic multilayer transverse by a current," Phys. Rev. vol. B 54, pp. 9353-9358, October 1996.

[3] J. Slonczewski, "Current-driven excitation of magnetic multilayers," J. Magn. Magn. Mater., vol. 159, pp. L1-L7, December 1996.

[4] M. Tsoi, A. G. M. Jansen, J. Bass, W.-C. Chiang, M. Seck, V. Tsoi, and P. Wyder, "Excitation of a Magnetic Multilayer by an Electric Current," Phys. Rev. Lett. vol. 80, pp. 4281-4284, May 1998.

[5] Z. Sun, "Current-driven magnetic switching in manganite trilayer junctions,” J. Magn. Magn. Mater., vol. 202, pp. 157162, December 1999.

[6] E. B. Myers, D. C. Ralph, J. A. Katine, R. N. Louie, and R. A. Buhrman," Current-Induced Switching of Domains in Magnetic Multilayer Devices," Science, vol. 285, pp. 867-870, October 1999.

[7] J. A. Katine, F. J. Albert, R. A. Buhrman, E. B. Myers, and D. C. Ralph, "Current-Driven Magnetization Reversal and SpinWave Excitations in $\mathrm{Co} / \mathrm{Cu} / \mathrm{Co}$ Pillars," Phys. Rev. Lett., vol. 84, 3148-3152, April 2000.

[8] T. Valet and A. Fert,"Theory of perpendicular magnetoresistance in magnetic multilayers," Phys. Rev., vol. B 48, pp. 7099-7104, April 1993.

[9] H. E. Camblong, P. M. Levy, and S. Zhang, "Electron transport in magnetic in homogeneous media," Phys. Rev., vol. B 51, pp. 16052-16072, June 1995.

[10] M. D. Stiles, "Oscillatory exchange coupling in $\mathrm{Fe} / \mathrm{Cr}$ multilayers," Phys. Rev., vol. B 54, pp. 14679-14685, November 1996.
[11] K. M. Schep, J. B. A. N. van Hoof, P. J. Kelly, G. E. W. Bauer, and J. E. Inglesfield, "Interface resistances of magnetic multilayers," Phys. Rev., vol. B 56, pp. 10805-10808, November 1997.

[12] A. Brataas, Y. V. Nazarov, and G. E. W. Bauer, "FiniteElement Theory of Transport in Ferromagnet-Normal Metal Systems," Phys. Rev. Lett., vol. 84, pp. 2481-2484, March 2000 .

[13] X. Waintal, E. B. Myers, P. W. Brouwer, and D. C. Ralph, "Role of spin-dependent interface scattering in generating current-induced torques in magnetic multilayers," Phys. Rev., vol. B62, pp. 12317-12327, May 2000.

[14] G.-M. Choi, B.-C. Min, K.-J. Lee, and D. G. Cahill, "Spin current generated by thermally driven ultrafast demagnetization," Nat. Commun., vol. 5, pp. 4334-4341, June 2014.

[15] A. Kirilyuk, A. V. Kimel, and T. Rasing, "Laser-induced magnetization dynamics and reversal in ferrimagnetic alloys," Rev. Mod. Phys., vol. 82, 2731-2784 September 2010.

[16] A. Kirilyuk, A. V. Kimel, and T. Rasing, "Laser-induced magnetization dynamics and reversal in ferrimagnetic alloys," Rep. Prog. Phys., vol. 76, pp. 026501-(1)-(5), May 2013.

[17] E. M. Lifshitz and L. P. Pitaevskii. Statistical Physics, Part 2, 3rd ed., Course of Theoretical Physics, vol. 9, Oxford: Pergamon, 1980.

[18] J. Xiao, G. E. W. Bauer, K.-C. Uchida, E. Saitoh, and S. Maekawa, "Theory of magnon-driven spin Seebeck effect," Theory of magnon-driven spin Seebeck effect," Phys. Rev, vol. B 81, pp. 214418(1)-(8), June, 2010.

[19] G. E. W. Bauer, E. Saitoh, and B. J. van Wees, "Spincaloritronics," Nat. Mater., vol. 11, pp. 391-399, April 2012.

[20] B. Koopmans, J. J. M. Ruigrok, F. Dalla Longa, andW. J. M. de Jonge, "Unifying Ultrafast Magnetization Dynamics," Phys. Rev. Lett., vol. 95, pp. 267207, December 2005.

[21] J. Walowski, G. Müller, M. Djordjevic, M. Münzenberg, M. Kläui, C. A. F. Vaz, and J. A. C. Bland, "Energy Equilibration Processes of Electrons, Magnons, and Phonons at the Femtosecond Time Scale,” Phys. Rev. Lett., vol. 101, pp. 237401, December 2008.

[22] A. Brataas, A. D. Kent, and H. Ohno, "Current-induced torques in magnetic materials," Nat. Mater., vol. 11, 372-381, December 2012.

[23] E. G. Tveten and A. Brataas, "Electron-magnon scattering in magnetic heterostructures far out of equilibrium," Phys. Rev., vol. 92, pp. 180412-(1)-(5), November (2015).

[24] L. Berger, "Exchange interaction between ferromagnetic domain wall and electric current in very thin metallic films," J. Appl. Phys., vol. 55, pp. 1954-1956, April 1984.

[25] C. W. J. Beenakker, "Random-matrix theory of quantum transport,” Rev. Mod. Phys., vol. 69, pp. 731-808, July 1997.

[26] K. B. Hathaway and J. R. Cullen, "A free electron model for the exchange coupling of ferromagnets through paramagnetic metals," J. Magn. Magn. Mater., vol. 104-107, pp. 1840-1842, November 1992.

[27] J. C. Slonczewski, "Mechanism of interlayer exchange in magnetic multilayers," J. Magn. Magn. Mater., 126, 374-379, May 1993. 
[28] P. Bruno, "Theory of interlayer magnetic coupling," Phys. Rev., vol. B 52, 411-439, July 1995.

[29] D. M. Edwards, F. Federici, J. Mathon, and A. Umerski, "Selfconsistent theory of current-induced switching of magnetization," Phys. Rev., vol. B 71, pp. 054407-(1)-(16), February 2005.

[30] A. Brataas, Yu. V. Nazarov, and G. E. W. Bauer, "FiniteElement Theory of Transport in Ferromagnet-Normal Metal Systems," Phys. Rev. Lett., vol. 84, pp. 2481-2484, March 2000 .

[31] P. W. Brouer and C. W. J. Beenakker, "Diagram method of integration over the unitary group, with applications to quantum transport in mesoscopic systems," J. Math. Phys., vol. 37, pp. 4904-4934, July 1996.

[32] J. Slonczewski, "Excitation of spin wave by electric current," Magn. Magn. Mater., vol. 195, pp. L261-L268, September 1999.

[33] E. Beaurepaire, J.-C. Merle, A. Daunois, and J.-Y. Bigot, "Ultrafast Spin Dynamics in Ferromagnetic Nickel," Phys. Rev. Lett., vol. 76, pp. 4250-4253, May 1996.

[34] J.-Y. Bigot, M. Vomir, and E. Beaurepaire, "Coherent Ultrafast magnetism induced by femtosecond laser pulses," Nat. Phys., vol. 5, pp. 515-520, May (2009).

[35] A. J. Schellekens and B. Koopmans, "Microscopic model for ultrafast magnetization dynamics of multisublattice magnets," Phys. Rev., vol. B 87, pp. 020407(1)-(5) (2013).

[36] A. Eschenlohr, M. Battiato, P. Maldonado, N. Pontius, T. Kachel, K. Holldack, R. Mitzner, A. F"ohlisch, P. M. Oppeneer, and C. Stamm, "Ultrafast spin transport as key to femtosecond demagnetization,” Nat. Mater., vol. 12, pp. 332-336, January 2013.

[37] C. Illg, M. Haag, and M. Fähnle, "Ultrafast demagnetization after laser irradiation in transition metals: $A b$ initio calculations of the spin-flip electron-phonon scattering with reduced exchange splitting," Phys. Rev., vol. B 88, pp. 214404(1)-(5) August 2013.

[38] Y. Zhang, T.-H. Chuang, K. Zakeri, and J. Kirschner, "Relaxation Time of Terahertz Magnons Excited at Ferromagnetic Surfaces," Phys. Rev. Lett., vol. 109, pp. 087203(1)-(5) August 2012.
[39] Y. Tserkovnyak, A. Brataas, G. E. W. Bauer, and B. I. Halperin, "Nonlocal magnetization dynamics in ferromagnetic heterostructures,” Rev. Mod. Phys., vol. 77, pp. 1375-1421, October 2005.

[40] S. A. Bender and Y. Tserkovnyak, "Interfacial spin and heat transfer between metals and magnetic insulators," Phys. Rev., vol. B 91, pp. 140402(1)-(5), April 2015.

[41] A.-P. Jauho, N. S. Wingreen, and Y. Meir, "Time-dependent transport in interacting and noninteracting resonanttunneling systems," Phys. Rev., vol. B 50, pp. 55285544, August 1994.

[42] B. Y. Mueller, A. Baral, S. Vollmar, M. Cinchetti, M. Aeschlimann, H. C. Schneider, and B. Rethfeld, "Feedback Effect during Ultrafast Demagnetization Dynamics in Ferromagnets," Phys. Rev. Lett., vol. 111, pp. 167204(1)-(5) 2013.

[43] J. Barker, U. Atxitia, T. A. Ostler, O. Hovorka, O. ChubykaloFesenko, and R. W. Chantrell, Sci. Rep., vol. 3, pp. 3262(1)-(5) (2013).

[44] Y. Tserkovnyak, E. M. Hankiewicz, and G. Vignale, "Transverse spin diffusion in ferromagnets," Phys. Rev., vol. B79, pp. 094415(1)-(11), March 2009.

[45] R. Meservey and P. M. Tedrow, Phys. Rev. Lett., vol. 41, pp. 805 (1978).

[46] U. Atxitia, O. Chubykalo-Fesenko, J. Walowski, A. Mann, and M. M"unzenberg, "Evidence for thermal mechanisms in laserinduced femtosecond spin dynamics," Phys. Rev., B 81, pp. 174401(1)-(8), May 2010.

[47] A. Weber, F. Pressacco, S. Gunther, E. Mancini, P. M. Oppeneer, and C. H. Back, "Ultrafast demagnetization dynamics of thin $\mathrm{Fe} / \mathrm{W}(110)$ films: Comparison of time- and spin-resolved photoemission with time-resolved magnetooptic experiments," vol. 84, pp. 132412(1)-(5), October 2011.

[48] Y. Liu, Z. Yuan, R. J. H. Wesselink, A. A. Starikov, and P. J. Kelly, "Interface Enhancement of Gilbert Damping from First Principles Phys. Rev. Lett., vol. 113, pp. 207202(1)-(5), November 2014. 